\title{
Cognitive Selection Mechanism Performance in IEEE 802.11 WLAN
}

\author{
W. Hashim, A. F. Ismail, N. A. Abd Ghafar, and S. Dzulkifly
}

\begin{abstract}
Recent growth in Wireless Local Area Network (WLAN) usage has generated considerable interest in the establishment of the IEEE 802.11 WLAN standards. WLAN has been developed with the capability to handle tremendous growth of data traffic and therefore have been proliferating worldwide. The influencing factors and the parameters involved prior choosing which access point (AP) to connect with are to be carefully evaluated and dealt with in the process of WLAN deployment. Properly designed selection mechanisms that able to provide not only radio coverage but also a good internet connection speed are in turn expected to be able to improve the Quality of Service (QoS). In this paper, a new approach for the AP selection in IEEE 802.11 is proposed. The new technique takes into account internet connection speed in contrast to the classic approach that solely based on the Received Signal Strength (RSS). A heuristic selection model was developed namely Evaluative AP Selection which used LINUX Bash script programming whilst the internet connection speed test was ran by means of PHP script. The results for the selection were evaluated respectively to see the variation in selection pattern when compared to the default selection algorithm. The time interval during the scanning and selection process was also analysed in the investigation to further identify the performance of the model. The enhancement reaped from the developed selection algorithm is expected to bring benefit to different user at various places with diverse requirements.
\end{abstract}

Index Terms-IEEE 802.11, WLAN, selection mechanism, connection speed, received signal strength, QoS.

\section{INTRODUCTION}

In today's fast-paced, technologically-driven world, there has been a widespread growth in the usage of Wireless Local Area Network (WLAN). WLAN is a communication technology that provides connectivity to wireless devices within a limited geographic area using radio waves as the transmission medium [1].

There are many choices of wireless LAN access points (AP) available in the market and most of the products are conform to the IEEE 802.11a, 802.11b, 802.11g and $802.11 \mathrm{n}$ wireless standards collectively known as Wi-Fi technology.

The reason why such is becoming the connection mode of choice is due to its reduced equipments price i.e. the wireless APs and the wireless network cards. The decreasing

Manuscript received February 8, 2013; revised April 17, 2013. This work was supported in part by the Malaysia Ministry of Science, Technology and Innovation, grant no SF-01-03-04-0018.

W. Hashim, S. Dzulkifly, and N. A. Abd Ghafar are with the Malaysian Institute of Microelectronic Systems (MIMOS Berhad), Kuala Lumpur, 57000 Malaysia (e-mail: wahidah.hashim@mimos.my).

A. F. Ismail is with the Electrical Engineering Department, University of International Islamic, Kuala Lumpur, 53100 Malaysia (e-mail: af_ismail@iium.edu.my). cost of fixed broadband services, including digital subscriber line (DSL) and fiber-to-the-home (FTTH) as well as the increases in WLAN data rates are among main reasons for the mass deployment of IEEE 802.11 WLAN over the last years. Universities, schools and many public places including food outlets are now providing Wi-Fi connectivity. Internet users are now becoming more dependent on portable laptops, PDAs, iPads, smart phones and other technological gadgets to get connected to the internet.

WLAN users or WLAN stations (STA) need to be connected to the AP with the best connection among the entire available units in order to experience the best Quality of Service (QoS) [2], [3]. By doing so, utilization of network resources can be fully achieved. Thus, the issue rises on what would be the optimal decision of AP selection mechanism by which performance metrics need to be considered.

Conventionally, an STA will be connected to the AP whose signal strength is the highest after performing the scanning (active or passive). This classic approach of AP selection mechanism that relies solely on signal strength had been criticized in [4] and [5]. The method ignores important parameters that matter to the STA and thus likely to degrade the network performance as well as the link quality. Moreover, this so-called strongest signal strength (SSS) policy can create high concentrations in one single AP. The other APs that are placed at slightly further distances from the STAs will not be connected and as the results they are underutilized. . It is noted that the throughput of each STA proportionally decreases with the number of STAs connected to the same AP as explained in [6], thus a highly concentrated AP inflicts severe effects e.g congestion to the rest of the network mentioned by [7] and [8].

Authors in [7], [9], [10] and [11] proved that the traditional approach in selecting an AP with the highest signal strength has known to cause the low network performance and can lead to imbalances in load per AP. Several critical parameters have to be put into consideration before an STA is to be connected to an AP. Among the parameters are maximum theoretical data rates, actual average data rate, time interval of channel occupation, AP traffic load and channel interference.

This paper proposed a new AP selection mechanism design capable of alleviating the current predicaments. The mentioned critical parameters will be exploited in the attempt to improve user's satisfaction. The rest of the paper is structured as follows: Section II provides a summary of the default AP selection protocol. Section III explains a brief description of the evaluative selection mechanism and Section IV highlights the experimental results gained. The conclusions drawn from this performance comparison study are summarized in Section V. 


\section{Default AP Selection Protocol}

A station (STA) default access process involves three steps: active/passive scanning, authentication and association [12]. In default setting of current implementation, prior associating with an AP, a STA will exercise scanning either active or passive scanning. By passive scanning, the beacon frames are periodically sent by all the available APs containing information about the AP including service set identifier (SSID), supported data rates and received signal strength (RSS). Active scanning on the other hand allows STA to receive immediate response from AP without waiting for the periodic beacon frames transmission in which a STA broadcasts a probe frame and all APs within range respond with a probe response [13]. After the scanning process, a STA will be connected to the AP which has the strongest signal strength according to the RSS. This method of relying on the signal strength has proven to lead to low network performance and can cause load imbalances in a particular $\mathrm{AP}$, as verified in [14].

Most popular operating systems (OS) such as Windows, Macintosh and Linux use essentially the same policy to guide the user to select an AP to associate with when more than one AP is available. For example, when a STA first boots or wakes from hibernation, it scans for all available APs. If the STA finds an AP which is previously saved as preferred AP, it will automatically associates to it. Otherwise, the user will have to manually connect to their preferred AP based on the RSS values. It is important to take note that each STA can associate with only one AP, although an AP can associate with multiple STAs.

\section{Selection Mechanism}

\section{A. Evaluative Selection}

The evaluative selection used the method of specifying the threshold value as the trigger for the next selection step. Denoted as Fig. 1, below is the flowchart of the overall selection process for evaluative model. This model starts off with connecting to the default connection while inquiring user whether or not they want to perform the scanning process.

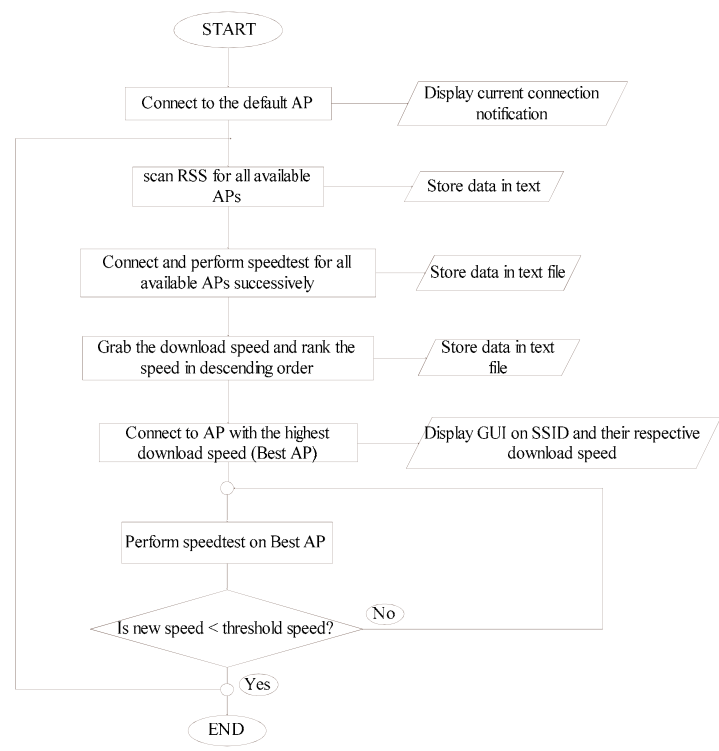

Fig. 1. Flowchart of evaluative selection mechanism.
When user chooses to perform the scanning, the overall evaluative selection model commences its procedure. The initial part of the evaluative selection model also involves connecting and scanning all the APs. The connection speed data obtained from the scanning process are stored and ranked based on the highest value. Once all the connection speeds have been ranked; the second fastest connectivity is established as the threshold value. The threshold value obtained is going to be the set off point for initiating new overall scanning.

This means that when the new value is less than the threshold value, new sequential scanning of all connections will be carried out once again. Following the ranking process conducted earlier, good connectivity is selected based on the highest download speed value.

The connection is then continuously monitored within a specified period of time, which in this case 30 minutes was selected. For every 30 minutes, the new download speed of the connection is assessed and compared to the threshold download speed. If the new speed is greater than the threshold speed, user will remain at the current connection.

On the other hand, when the new speed is less than the threshold speed, new scanning process for all APs will be started and new connection will be based on the new highest speed ranked.

\section{B. System Architecture}

Fig. 2 illustrates the design scenario of the network selection algorithm. Three different APs namely AP1, AP2 and AP3 were installed at different places in an office where each one has no encryption. Each AP was connected to the Ethernet connection as the backhaul and thus provides sufficient internet connectivity to run the speed test on each.



Fig. 2. Design scenario of network selection.

An experimental laptop acted as a user to successively run the speed test on each AP and will then processed the data gained in order to intelligently select which AP to connect to.

\section{Experimental Setup}

The test bed setup consisted of 2 main components which were user's terminal and APs as shown in Fig. 3. The 3 APs used in this study AP1, AP2 and AP3 have been configured in LINUX programming language with the Ethernet connection as the backhaul connection.

The placement of AP1, AP2 and AP3 are illustrated as in Fig. 4. The distance of each AP from the user's terminal varies accordingly. AP1 was the nearest at approximately $1 \mathrm{~m}$ away and AP3 located farthest at approximately $10 \mathrm{~m}$ from the user's terminal. The nearest AP to the user will give the highest RSS value recorded at the terminal. The idea behind the various displacements of the APs is to prove 
that the highest RSS value did not warrants fastest internet connection speed. Even though AP1 has the highest RSS value, there is no assurance that it will get connected since another parameter which is the internet connection speed will be taken into consideration in the proposed procedure.



Fig. 3. Experimental test bed setup.

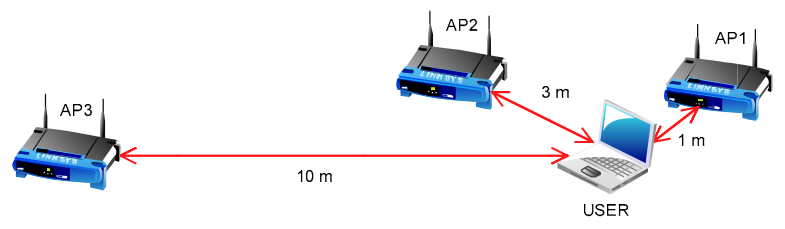

Fig. 4. Location of APs with respect to user's terminal.

\section{System Parameters}

The justification for the use of the custom-built AP instead of the normal stand alone AP is because the ability to self write and modify the management software. The AP specifications are summarized in Table I below.

TABLE I: SYSTEM PARAMETERS

\begin{tabular}{|c|c|c|c|}
\hline \multicolumn{3}{|c|}{ Specifications } \\
\hline Attributes & AP1 & AP2 & AP3 \\
\hline SSID & $2.462 \mathrm{GHz}$ & $2.462 \mathrm{GHz}$ & $2.462 \mathrm{GHz}$ \\
\hline Frequency & B0:48:7A:F7: & $5 \mathrm{C}: \mathrm{D} 9: 98: 45:$ & F0:7D:68:C4: \\
BAC Address & BA: 5 & D & 97 \\
\hline Max. transmit bit rate & $54.0 \mathrm{Mbps}$ & $54.0 \mathrm{Mbps}$ & $54.0 \mathrm{Mbps}$ \\
\hline Channel & 11 & 11 & 11 \\
\hline
\end{tabular}

\section{E. Data Trace and Analysis}

To execute the algorithm, the programming part was done at LINUX terminal by exploiting the bash script as well as the PHP script. The programming scripts were developed in such a flow that it will initially seized the RSS value of each AP. A command line was run from the terminal to compute the RSS parameter. The beacon frame of the AP was automatically generated and stored in a file that contains the MAC address, SSID, carrier frequency, transmit packet bytes, receive packet bytes, RSS and transmit bit-rate. The bash scripts resumed by successively ran the connection speed test for each AP starting with the AP with the highest RSS by calling the PHP script.

The PHP script provides the critical information such as the ping (latency), average download speed and the average upload speed. It functions entirely over HTTP to measure the maximum compatibility of the deployed AP. The ping value (latency) indicates the time it takes to get a response from the selected server when the HTTP request was being sent. In this paper, the same server in Kuala Lumpur region was used to ensure the uniformity of the sampling process. The download speed is regarded as the downloading of a binary file from the web server to the client, and the download speed is measured to estimate the connection speed. Based on this result, a specific amount of data can be selected for download in a real test. The main purpose is to determine the appropriate amount of data that can be downloaded in 10 seconds, ensuring enough data gained for an accurate result but do not involve an extended period of time.

The upload speed on the other hand is determined when a small amount of random data is generated in the client and sent to the web server to estimate the connection speed. Based on this result, an appropriately sized chunk of randomly generated data is selected for uploading purposes. The upload test data are then performed in chunks of uniform size data to the server-side. They are sorted by speed, and the fastest half is averaged to eliminate anomalies.

\section{EXPERIMENTAL RESULTS}

The results for the evaluative selection mechanism were analyzed and plotted in graph accordingly. In the selection model, 15 numbers of laps took place with the interval of 30 minutes for each lap. The AP selected by the model along with their individual average download speed was then represented in graph form as in Fig. 5 below.

Referring to Fig. 5, the download speed of the currently connected AP was spotted to be below the threshold value during lap 5. This evaluative selection will perform a new speed test and thus connect to the AP with the highest speed value. This is to ensure that the user will have the most upto-date and accurate information on the APs speed attribute. TS denotes as threshold speed value.

A new connection speed test was run during Lap 6 to choose the AP with the highest connection speed. The second highest value of $1.09 \mathrm{Mbps}$ which belongs to AP2 was set to be the new threshold speed value, denoted as TS2 in the graph. The connection at AP1 persisted until then again the current speed value went below the threshold at Lap 9. As a result of the connection speed test, AP2 was chosen to be connected with. The algorithm continued at every 30 minutes when the current speed value was spotted to be below threshold (TS3) during Lap 12. AP1 was connected at Lap 13 as it had the highest connection speed value and the connection at this AP endured until the final lap.

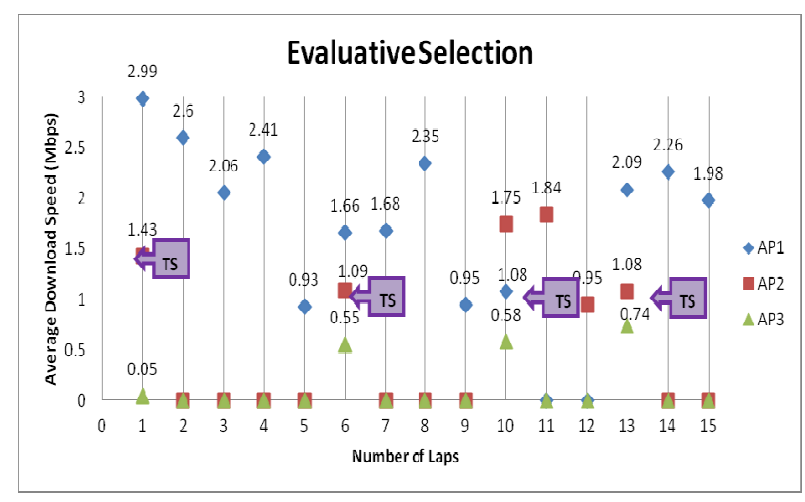

Fig. 5. AP selection results of evaluative selection mechanism.

To further investigate the performance of the developed selection models, the results obtained were then evaluated from two different prospects. For the first part, the 
evaluative selection data rate during one complete cycle i.e. 15 laps was measured and made compare to the data rate of the default AP selection as displayed in Fig. 6. It is to be noted that the default AP selection chose AP1 as the AP of choice for the whole duration.

The second part involved the assessment made on the evaluative selection algorithm with the default selection in terms of the percentage difference of the averaged data rate. From Fig. 7, the averaged data rate of both selections was calculated during the 15 laps interval. The average data rate value was $1.97 \mathrm{Mbps}$ and $1.59 \mathrm{Mbps}$, for the evaluative AP selection and the default AP selection respectively. This remarks an improvement of $23.9 \%$ in data rate when using the evaluative selection algorithm as compared to the default AP selection.

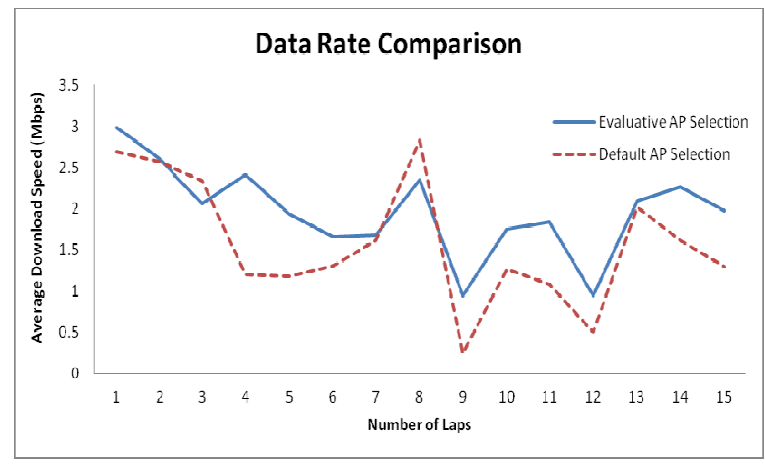

Fig. 6. Data rate comparison with the default AP selection.

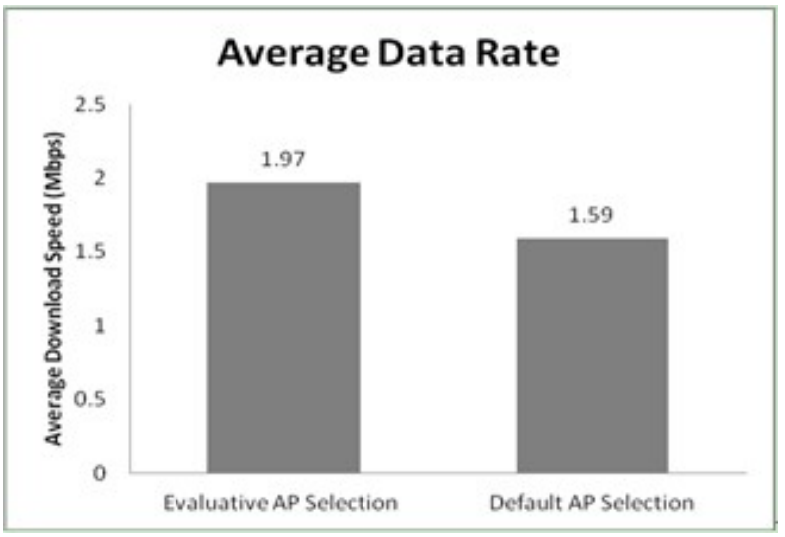

Fig. 7. Average data rate comparison with the default AP selection.

\section{CONCLUSION}

The evaluative selection model had shown performance improvement of $23.9 \%$ in terms of average data rate when compared with the default AP selection which relies solely on the RSS. This model is recommended to be employed by user who requires highest connection speed at real time. The limitation of this model involves disconnection for a specific period of time while the algorithm performs scanning for all APs.

\section{ACKNOWLEDGMENT}

The studies are part of deliverables to Ministry of Science, Technology and Innovation of Malaysia. The reported research findings are part of the main research funded under Science fund. It is a collaboration project under supervision of MIMOS Berhad incorporating researchers from International Islamic University of Malaysia (IIUM).

\section{REFERENCES}

[1] M. Haidar, "Load distribution and channel assignment in IEEE 802.11 wireless local area networks," PhD. dissertation, Technology University of Arkansas, 2007.

[2] K. Sundaresan and K. Papagiannaki, "The need for cross-layer information in access point selection algorithms," in Proc. the 6th ACM SIGCOMM Conference on Internet Measurement, pp. 257-262, 2006.

[3] H. Wu, K. Tan, Y. Zhang, and Q. Zhang, "Proactive scan: fast handoff with smart triggers for 802.11 wireless LAN," INFOCOMM '07, 2007, pp. 749-757.

[4] E. Ghazisaeedi and S. Zokaei, "A method for access point selection in 802.11 networks," in Proc. First International Conference on Networked Digital Technologies, NDT '09, 2009, pp. 447-451.

[5] A. J. Nicholson, Y. Chawathe, Y. M. Chen, B. D. Noble, and D. Wetherall, "Improved access point selection," Mobisys '06, 2006, pp. 233-245.

[6] Y. Fukuda, T. Abe, and Y. Oie, "Decentralized access point selection architecture for wireless LANs," in Proc. Wireless Telecommunications Symposium, 2004, pp. 137-145.

[7] A. Balachandran, G. M. Voelker, P. Bahl, and V. Rangan, "Characterizing user behavior and network performance in a public wireless LAN," in Proc. ACM SIGMETRICS '02, 2002, pp. 195-205.

[8] Y. Bejerano, H. S. Jae, and L. Li, "Fairness and load balancing in wireless LANs using association control," IEEE/ACM Transactions on Networking, vol. 15, No. 3, pp. 560-573, 2007.

[9] D. Tang and M. Baker, "Analysis of a local-area wireless network," in Proc. ACM Mobicom '00, pp. 1-20, 2000.

[10] D. Tang and M. Baker, "Analysis of a metropolitan-area wireless network," in Proc. ACM Mobicom '99, pp. 12-23, 1999.

[11] D. Kotz and K. Essien, "Analysis of a campus-wide wireless network," in Proc. the $8^{\text {th }}$ Annual International Conference on Mobile Computing and Networking, pp. 107-118, 2002.

[12] T. C. Ling, J. F. Lee, and K. P. Hoh, "Reducing handoff delay in WLAN using selective proactive context caching," Malaysian Journal of Computer Science, vol. 23, no. 1, pp. 49-59, 2010.

[13] V. Garg, Wireless Communications and Networking, in Elsevier Science, 2010, pp. 746-748.

[14] M. F. Tusyuz and H. A. Mantar, "Access point selection for improving the voice quality and overall throughput in wireless LANs," in Proc. ACM Mobicom '99, 2010, pp. 12-23.

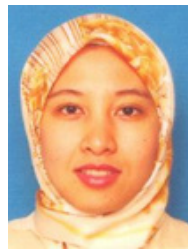

W. Hashim received his bachelor degree in information technology, business management and language from University of York, UK in 1999. She then pursued her MSc in Multimedia Technology at University of Bath, $\mathrm{UK}$ in 2001. She completed her $\mathrm{PhD}$ studies from King's College London, UK in 2008 in the field of Telecommunication Engineering. She is currently works at MIMOS Berhad wireless communication cluster as a staff researcher since 2009. Apart from her main task in doing research in cognitive radio, WLAN, OFDM, space-time coding, MIMO systems and wireless system, she is actively involved in the development of Malaysia technical specification, standard and guidelines of wireless terminal devices, International Mobile Telecommunication (IMT) systems and wireless sensor network for Malaysia Communications and Multimedia Commission (MCMC). She has occasionally represented Malaysia at International Telecommunication Union (ITU) working party 5D meeting for IMT-Advanced technology. Dr. Wahidah has published several publications and filed several patents on her research findings. She is a member of the IEEE.

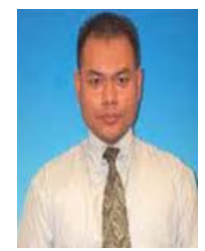

A. F. Ismail is currently serving as a lecturer at the Department of Electrical and Computer Engineering, Faculty of Engineering, International Islamic University Malaysia. He completed his bachelor degree studies in Electrical Engineering at Gannon University, Pennsylvania, USA with Cum Laude Latin honors. He holds MSc in Telecommunications and Information Systems from University of Essex, UK and $\mathrm{PhD}$ in Electronics from University of Bath, UK. His research interests include millimeter and microwave propagation studies, development of active and passive target tracking algorithms and Cognitive Radio (CR) applications. $\mathrm{He}$ is a registered Professional Engineer with Board of Engineering Malaysia and also a senior member of the IEEE. 




N. A. Abd Ghafar received the B.Eng (Hons) in Communication Engineering from International Islamic University of Malaysia in 2006. She is currently a graduate research assistant at Wireless Communications Cluster, MIMOS Berhad since 2011 and also a M.Sc. candidate in Communication Engineering, Department of Electrical and Computer Engineering, International Islamic University of Malaysia under the supervision of Assoc. Prof. Ir. Dr. Ahmad Fadzil Ismail. Her research interests are wireless communication particularly on the network selection management for the next generation wireless communication network.

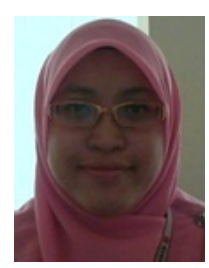

S. Dzulkifly obtained her bachelor's degree in Communication Engineering from International Islamic University Malaysia (IIUM). She is currently pursuing Master's degree in Communication Engineering at the same university under the supervision of A. F. Ismail. At the same time, she works as research assistant at Malaysian Institute of Microelectronic Systems (MIMOS Berhad) on a research funded by Ministry of Science, Technology and Innovation (MOSTI). Throughout her period working in MIMOS Berhad, she had assisted in the related research works and co-authored several conference paper. Her research interest includes wireless broadband technologies and artificial intelligent/cognitive selection system. 